\title{
Urinary Retention Secondary to Listeria Meningitis
}

\author{
Kohei Fujita ${ }^{1}$, Tomotaka Tanaka ${ }^{1}$, Syoichiro Kono ${ }^{1}$, Hisashi Narai ${ }^{1}$, \\ Nobuhiko Omori ${ }^{1}$, Yasuhiro Manabe ${ }^{1}$ and Koji $\mathrm{Abe}^{2}$
}

\begin{abstract}
We report a rare case of urinary retention secondary to Listeria meningitis. A 90-year-old woman presented with high fever, nausea, diarrhea, and incontinence of urination and feces. Lumbar puncture was performed. The total cell number of the cerebrospinal fluid (CSF) was elevated indicating that the glucose level was decreased. A CSF culture and a blood culture revealed Listeria monocytogenes (L. monocytogenes). We treated this bacterial meningitis with antibiotic medicine. One month after onset, stiff neck and laboratory data greatly improved and only urinary retention continued. Lumbar magnetic resonance imaging (MRI) showed no responsible lesions for the urinary retention. She received urological examination for urinary retention and was diagnosed with a neurogenic bladder. Two months later, she was able to walk after rehabilitation. However, the urinary retention continued despite urological therapy. We are not aware of descriptions on urinary retention resulting from bacterial meningitis in the recent literature. This is a rare case of prolonged urinary retention caused by bacterial meningitis.
\end{abstract}

Key words: Listeria meningitis, urinary retention, bacterial meningitis

(Inter Med 47: 1129-1131, 2008)

(DOI: 10.2169/internalmedicine.47.0985)

\section{Introduction}

Listeria monocytogenes (L. monocytogenes) is isolated from soil, milk, and raw meat, and often causes outbreaks of food poisoning (1). The incidence rate of listeriosis in the world is 0.2 to 7.4 per one million (2). It is well known that infants and the elderly are easily infected. Listeria meningitis accounts for $3 \%-4 \%$ of all cases of adult bacterial meningitis, and has many types of acute complications as compared to other types of meningitis (3-7). However, there has been no description of urinary retention in the recent literature (8). Here, we report a rare case of urinary retention secondary to Listeria meningitis.

\section{Case Report}

A 90-year-old woman with a history of iron-deficiency and anemia complained of fever, nausea, diarrhea, and incontinence of urination and feces with difficulty in ambulation. She was admitted to our hospital on day 3 after the on- set. Her general physical examination showed no abnormalities. On neurological examination, her cranial nerves were intact. Motor examination revealed 4/5 strength in both lower extremities. Deep tendon reflexes were normal, and no pathological reflexes, ataxia or sensory disorders were noted. She had a stiff neck, but Kernig's sign was absent. Laboratory data showed a white blood cell (WBC) count of $7,200 / \mu \mathrm{L}$, and C-reactive protein (CRP) count of $11.24 \mathrm{mg} /$ dL. At lumbar puncture, the cerebrospinal fluid (CSF) pressure was $180 \mathrm{mmH}_{2} \mathrm{O}$. Cell count was $939 / \mu \mathrm{L}$ (neutrophil 384, lymphocyte 555). The CSF protein concentration of $277 \mathrm{mg} / \mathrm{dL}$ was elevated and glucose level of $52 \mathrm{mg} / \mathrm{dL}$ (blood glucose $172 \mathrm{mg} / \mathrm{dL}$ ) was decreased. Cranial computed tomography (CT) showed no parenchymal abnormality. Gram staining of the CSF showed few gram-positive rods. From the time of admission, she was treated with ceftriaxone $2 \mathrm{~g} /$ day as empirical therapy for bacterial meningitis. CSF culture and blood culture revealed L. monocytogenes. We changed ceftriaxone for ampicillin $2 \mathrm{~g} / \mathrm{day}$ and gentamicin $80 \mathrm{mg} /$ day. A urinary catheter was inserted on the third day because of urinary retention after admission.

${ }^{1}$ Department of Neurology, National Hospital Organization Okayama Medical Center, Okayama and ${ }^{2}$ Department of Neurology, Graduate School of Medicine and Dentistry, Okayama University, Okayama

Received for publication February 4, 2008; Accepted for publication March 25, 2008

Correspondence to Dr. Yasuhiro Manabe, ymanabe@okayama3.hosp.go.jp 

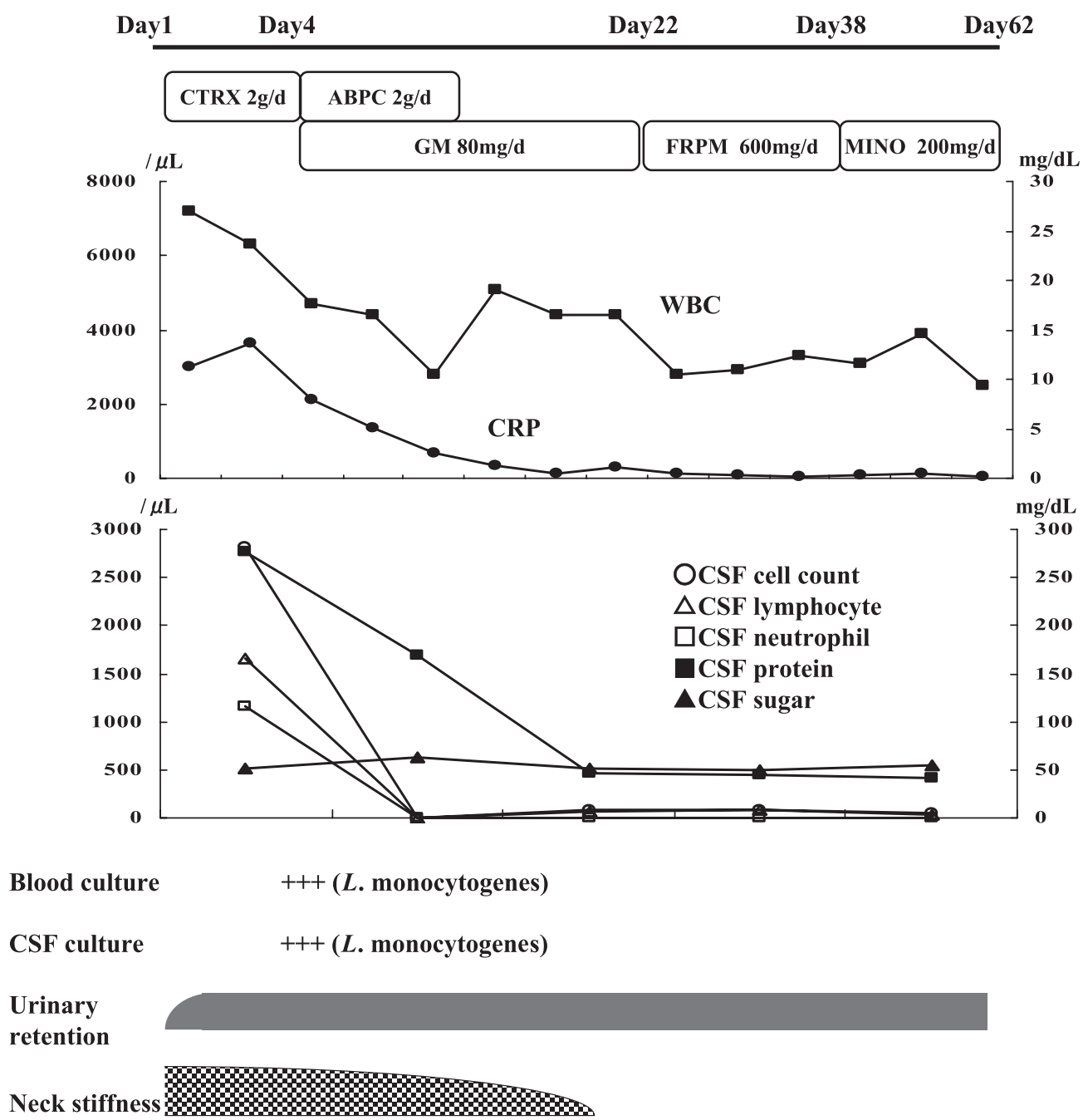

Figure 1. Clinical course of the patient. ABPC: ampicillin, CRP: c-reactive protein, CSF: cerebrospinal fluid, CTRX: ceftriaxone, FRPM: faropenem, GM: gentamicin, L. monocytogenes: Listeria monocytogenes, MINO: minocycline, WBC: white blood cell.

Laboratory data on the eighth day showed pancytopenia. In consideration of a side effect from ampicillin, only gentamicin was used. Laboratory data on the 22nd day showed a WBC count of $2,800 / \mu \mathrm{L}$, and a CRP count of $0.46 \mathrm{mg} / \mathrm{dL}$. We changed her medication from intravenous use of antibiotic medicine to internal use of faropenem $600 \mathrm{mg} /$ day. Stiff neck and laboratory data greatly improved and only urinary retention continued. However, her superficial and deep sensations were decreased in her bilateral distal lower extremities. The distribution of hypesthesia was corresponding to $\mathrm{S}$ 2-3 segments. The site of lesion was thought to be in the sacral region. Lumbar magnetic resonance imaging (MRI) was performed to check for organic factors of urinary retention, but there was no lesion responsible for the urinary retention. On the 24th day of illness, she underwent a urological examination for urinary retention, and was diagnosed with a neurogenic bladder. She had slight micturition and overflow incontinence. Postvoid recidual volume was 300 $\mathrm{mL}$. Combined use of medical therapy with distigmine 10 $\mathrm{mg} /$ day and urapidil $30 \mathrm{mg} /$ day was started. In spite of use of faropenem for 17 days, CRP did not return to negative in laboratory data and the total cell number of CSF did not decrease on the 38th day of illness. Thus, internal use was replaced with minocycline $200 \mathrm{mg} /$ day. CRP turned negative and the total cell number of CSF finally decreased to $14 / \mu \mathrm{L}$ (neutrophil 1, lymphocyte 13) on the 45th day of illness. Although urinary retention remained and forced intermittent self-catheterization was used, she was able to walk after rehabilitation, and was discharged from the hospital on the 62 nd day of illness (Fig. 1).

\section{Discussion}

This patient almost completely recovered from Listeria meningitis after two months; however, urinary retention remained. Urinary retention caused by aseptic meningitis is well known, and includes Elsberg syndrome, which occurs with herpes virus meningitis. The recent literature does not contain descriptions on urinary retention resulting from bacterial meningitis, apart from those with tubercular meningitis (8). Of 46 reports we found after a literature search, 42 cases involved urinary retention due to aseptic meningitis, 3 
involved tubercular meningitis, and 1 was a result of spinal cord abscess. Precentral gyrus of the brain cortex, pons, sacral cord, and centrifugal nerves from the sacral cord were believed to exist in the deficit regions, which caused urinary retention. In the present case, the sacral cord and centrifugal nerves were suspected as the deficit regions because the patient had sensory disturbance at the sacral level, a decrease of micturition and remaining urine impression, without a serious brain condition, such as conscious deficit, agnosia, or aphasia. In cases of urinary retention due to the aseptic meningitis in our literature search, almost all cases improved within one month, except for one case that required six months, and no cases reported sequelae. Cranial nerve deficit from bacteria is thought to be stronger than that from viruses; therefore, the prognosis of urinary retention caused by bacterial meningitis may be worse than that by aseptic meningitis. In conclusion, we report a rare case of prolonged urinary retention caused by bacterial meningitis.

\section{References}

1. Schlech WF, Lavigne PM, Bortolussi RA, et al. Epidemic listeriosis-evidence for transmission by food. N Engl J Med 308: 203206, 1983.

2. WHO. Human listeriosis. Bull World Health Organ 71: 259-260, 1993.

3. Kaplan SL, Kaufman BA, Hartman BJ, et al. Practice guidelines for the management of bacterial meningitis. Clin Infect Dis 39: 1267-1284, 2004.

4. Mylonakis E, Hohmann EL, Calderwood SB. Central nervous system infection with Listeria monocytogenes: 33 years' experience at a general hospital and review of 776 episodes from the literature. Medicine 77: 313-336, 1998.
5. Spanos A, Harrell FE, Durack D. Differential diagnosis of acute meningitis: an analysis of the predictive value of initial observations. JAMA 262: 2700-2707, 1989.

6. Van de Beek D, de Gans J, Spanjaard L, et al. Antibiotic guidelines and antibiotic use in adult bacterial meningitis in the Netherlands. J Antimicrob Chemother 49: 661-666, 2002.

7. Van de Beek D, de Gans J, Spanjaard L, et al. Clinical features and prognostic factors in adults with bacterial meningitis. N Engl J Med 351: 1849-1859, 2004.

8. Wein AJ, Kavoussi LR, et al. Campbell-Walsh Urology. 9th ed. Saunders, Philadelphia, 2007: 2011-2033.

(C) 2008 The Japanese Society of Internal Medicine http://www.naika.or.jp/imindex.html 